\title{
PROKLA-Redaktion
}

\section{Editorial: China auf dem Weg zum Kapitalismus}

I.

Auf einen großen Teil der westeuropäischen Linken, die sich in den 60er Jahren jenseits der traditionellen Parteien der Arbeiterbewegung im Protest gegen den Vietnamkrieg konstituierte, übte das Sozialismusmodell der Volksrepublik China eine eigenartige Faszination aus. China konnte anscheinend nicht nur eine bessere Entwicklungsbilanz aufweisen als die meisten kapitalistisch orientierten Länder der sog. 3. Welt; auch sein politisches System schien nicht ganz so verkrustet zu sein, wie das der Sowjetunion. Vor allem die 1966 einsetzende „Kulturrevolution" stieß bei der studentischen Linken Westeuropas auf zunehmende Begeisterung: hier wurden nicht nur hierarchische Strukturen kritisiert, anscheinend ging es auch um die Veränderung kultureller Werte als Voraussetzung einer kommunistischen Gesellschaft. Zwar blieb diese Begeisterung für das chinesische Modell in den 70er Jahren nur noch in den diversen maoistischen Parteigründungen erhalten; es brauchte aber noch einige Zeit bis sich die Einsicht durchsetzte, daß mit der Kulturrevolution in erster Linie die Parteiführung um Mao Zedong ihre Macht zu behaupten versuchte, die nach dem Desaster des „Großen Sprungs“ angeschlagen war.

Ziel des 1958 begonnenen „Großen Sprungs nach vorne“ war eine beschleu- nigte wirtschaftliche Entwicklung, die durch dezentrale und arbeitsintensive Produktion erreicht werden sollte: jedem Dorf seinen eigenen kleinen Stahlkocher. Gleichzeitig wurden riesige Volkskommunen geschaffen, die auf die weitgehende Auflösung der privaten Hauswirtschaft abzielten und die Massenmobilisierung für Großprojekte erleichtern sollte. Der „Große Sprung nach vorne“ endete in einer wirtschaftlichen Katastrophe mit ca. 30 Millionen Hungertoten.

Mit der Kulturrevolution sollten nun einerseits die „rechten“ Kritiker innerhalb der Partei ausgeschaltet, andererseits die Massen erneut mobilisiert werden. Während der Kulturrevolution wurden große Teile der Universitäten geschlossen und Intellektuelle massenhaft zur Arbeit auf dem Land gezwungen. Rote Garden und Revolutionskomitees ersetzten die hergebrachte Parteihierarchie und wurden Träger der von Mao propagierten revolutionären Ideen. Die Kulturrevolution ergriff zunächst Hochschulen und ländliche Gebiete und wurde dann auf die Fabriken ausgedehnt, um auch dort die traditionellen Hierarchien zu ersetzen. Der einzig stabile Faktor blieb das Militär, an dem die Kulturrevolution weitgehend vorbeiging. Die Kulturrevolution führte nicht nur zu einem erneuten wirtschaftlichen Desaster (einschließlich Hungerkatastrophen), sie brachte auch ein 
bis dahin ungekanntes Ausmaß von Gesetzlosigkeit, Willkür und Massenrepression mit sich, was sich tief ins kollektive Bewußtsein eingegraben hat. Es ist nicht zuletzt die traumatisierende Erfahrung der Kulturrevolution, welche noch heute sicherstellt, dass die Mehrheit der Bevölkerung loyal zu einer Partei- und Staatsführung steht, die zwar autoritär regiert und auf offene Kritik schnell mit Repression reagiert, die aber zugleich „Ordnung“ und „Sicherheit“" gewährleistet.

II.

Mit dem Tod von Mao Zedong im Jahre 1976, der seit der Gründung der Volksrepublik China im Jahre 1949 Vorsitzender der Kommunistischen Partei war, und seines Weggefährten, dem Ministerpräsidenten Zhou Enlai, im gleichen Jahr kam die Kulturrevolution endgültig zu ihrem Ende. Im Verlauf des nun einsetzenden Machtkampfes wurde die sogenannte „Viererbande” (zu der auch die Witwe Maos gehörte) sowie eine Reihe weiterer hochrangiger Parteimitglieder verhaftet, die „Reformer“ setzten sich durch. Im Dezember 1978 wurde auf dem 3. Plenum des XI. Zentralkomitees der Kommunistischen Partei Chinas der Beginn des ökonomischen Reformprozesses beschlossen, dem China bis heute folgt. Dieser Reformprozess ist eng mit Deng Xiaoping verbunden, der aus dem Machtkampf innerhalb der Kommunistischen Partei als unumstrittener Führer hervorging. Deng war schon Mitte der fünfziger Jahre Vize-Ministerpräsident und Mitglied des Politbüros; 1966 wurde er im Rahmen der Kulturrevolution als „Revisionist“ entmachtet und zum Fabrikarbeiter degradiert. Erst 1973 wurde er auf Drängen Zhou Enlais rehabilitiert und erhielt seine früheren Funktionen zurück.
Der 1978 einsetzende Reformprozeß blieb stets unter der Kontrolle der kommunistischen Partei, wodurch sich die chinesische Entwicklung erheblich von der Transformation in den osteuropäischen Ländern unterscheidet. Nach dem Kollaps von 1989/90 verloren dort die alten kommunistischen Führungen ihre Macht, an ihre Stelle traten westlich orientierte Parteien mit einem mehr oder weniger neoliberalen Credo (dem sich schließlich auch die meisten verbliebenen ex-kommunistischen Parteien anschlossen): Es wurde auf die Freisetzung der „Marktkräfte“ und den weitgehenden Rückzug des Staates aus der Wirtschaft gesetzt.

In China ist dagegen nicht nur keine Opposition zugelassen, der wirtschaftliche Reformprozeß dient hier der Machtsicherung der kommunistischen Partei nach innen und nach außen. Nach innen soll die Loyalität der Bevölkerung durch eine Erhöhung des Lebensstandards gesichert werden, wobei eine zunehmende materielle Ungleichheit nicht nur in Kauf genommen, sondern als Anreiz zu größerer Leistung durchaus positiv gesehen wird. Gleichzeitig sollte die ökonomische Entwicklung aber auch die Unabhängigkeit nach außen sichern, sah sich China in den 70er und 80er Jahren doch zwischen zwei imperialistischen Blöcken (dem der Sowjetunion und dem der USA) eingezwängt. Im Unterschied zur sowjetischen Führung blieb sich die chinesische stets bewußt, dass sie ein Entwicklungsland regierte, das sich immer in der Gefahr befand, zum Spielball übermächtiger Gegner zu werden - wie einst im Opiumkrieg. Die Macht abzugeben oder auch nur zu teilen, kam während des ganzen Reformprozesses für die chinesische Führung niemals in Frage und durch die politische und öko- 
nomische Entwicklung Osteuropas sieht sie sich bis heute in dieser Haltung bestärkt.

$\mathrm{Zu}$ welchem Ausmaß an Unterdrückung die chinesische Führung nach wie vor bereit ist, zeigt nicht nur ihre kompromißlose Repressionspolitik in Tibet und die Verfolgung aller „Dissidenten“. Wie wenig ihr nicht nur Menschen- und Bürgerrechte, sondern auch Menschenleben zählen, wurde bei der brutalen Niederschlagung der „Demokratiebewegung“ am 4. Juni 1989 deutlich, als Truppen des Militärs ein Massaker auf dem Platz des Himmlischen Friedens anrichteten. Die vor allem von Studenten und städtischen Intellektuellen getragene „Demokratiebewegung" blieb das einzige etwas breitere Aufbegehren gegen das Machtmonopol der kommunistischen Partei. Nach der Niederschlagung der Demokratiebewegung kam es zwar kurzzeitig zu einer Unterbrechung des Reformprozesses, aber schon 1991 wurde von Deng Xiaoping während seiner berühmten „Reise in den Süden” der Beginn einer neuen Reformwelle einschließlich der Öffnung nach außen angekündigt. In der Tat beschleunigte sich der Reformprozess dann in den neunziger Jahren, woran auch der Tod von Deng im Jahre 1997 nichts änderte.

Westliche Regierungen haben nach dem Massaker auf dem Platz des himmlischen Friedens zwar ihre Beziehungen zu China vorübergehend eingefroren und lauthals die Verletzung der Menschenrechte beklagt, inzwischen herrscht aber wieder Business as usual - geht es doch um einen der größten Märkte der Welt, der sich zunehmend nach außen öffnet, und bei dessen Erschließung soll das nationale Kapital schließlich nicht zu kurz kommen. Auch die Politik der rotgrünen Bundesregierung unterscheidet sich hier um kein Deut von ihrer konservativen Vorgängerin.

Dass die Opposition gegen die autoritäre chinesische Führung in der Bevölkerung praktisch nicht verankert ist, hat nicht nur mit den traumatischen Erfahrungen der Kulturrevolution zu tun, sondern auch mit dem - gemessen an quantitativen Kriterien - außerordentlichen Erfolg des chinesischen Reformprozesses. Der Index des realen Bruttoinlandsproduktes in China stieg von 100 im Jahre 1989 innerhalb von 10 Jahren auf über 250; das Jahrzehnt davor wies ähnliche Wachstumsraten auf. Mit dem enormen Wachstum des Sozialproduktes war ein kräftiger Anstieg des Pro-Kopf-Einkommens verbunden. Der Lebensstandard hat sich in China für alle Teile der Bevölkerung seit 1978 deutlich erhöht. Für die Steigerung des Pro-Kopf-Einkommens ist auch die Ende der siebziger Jahre beschlossene und administrativ hart durchgesetzte Ein-Kind-Politik verantwortlich. Im Vergleich dazu erlebte Indien mit rund $1 \mathrm{Mrd}$. Einwohnern das zweite große Land Asiens (China hat ca. 1,3 Mrd. Einwohner) während der vergangenen zwei Jahrzehnte ein deutlich höheres Bevölkerungswachstum und ein wesentlich geringeres Wachstum des ProKopf-Einkommens. Bis Ende der neunziger Jahre gab es in China auch eine weitgehende Vollbeschäftigung bei den Industriearbeitern, da der staatliche Unternehmenssektor aus politischen Gründen keine Entlassungen vornahm. Die hinter der Überbeschäftigung in staatlichen Unternehmen und öffentlichen Verwaltungen versteckte Arbeitslosigkeit war allerdings recht hoch. Auch gab es in China für Industriearbeiter in Staatsunternehmen sowie für Beschäftigte in der Administration ein vergleichsweise komfortables soziales Netz. Soziale Här- 
ten wurden in den ersten beiden Jahrzehnten des Reformprozesses weitgehend vermieden. Seit Ende der 90 Jahre sind jedoch Vollbeschäftigung in den Städten und das soziale Netz in der hergebrachten Form nicht mehr aufrechtzuerhalten. Die materielle Basis, auf der die Loyalität der Bevölkerung ruht, bekommt damit erste Risse.

III.

Der chinesische Transformationsprozess ist gradualistisch: ökonomische Reformen wurden nur in kleinen Schritten und in der Regel auch zunächst geographisch begrenzt durchgeführt. Der erste etwa fünfjährige Reformschritt konzentrierte sich auf die Landwirtschaft. Dort wurden Produktionskollektive aufgelöst und durch ein familienzentriertes Pachtsystem ersetzt. Nach der Einführung des Pachtsystems stieg die Lebensmittelproduktion schlagartig an. Allerdings hat sich die landwirtschaftliche Produktion seither nur wenig verändert. Die Produktivität verharrt trotz einer sprunghaften Erhöhung zwischen Ende der siebziger und etwa Mitte der achtziger Jahre auf einem niedrigen Niveau und nach wie vor sind die meisten Arbeitskräfte in der Landwirtschaft beschäftigt.

Die ersten Experimente im Bereich industrieller Produktion fanden in speziellen Zonen und/oder Unternehmen statt; die Mengenplanung für die staatlichen Unternehmen wurde während der achtziger Jahre langsam abgebaut. Gleiches gilt für die Liberalisierung der Preise. Die Privatisierung der Staatsunternehmen stand in den 80er Jahren nicht auf dem Reformplan. Zugelassen wurden allerdings Neugründungen von Unternehmen, die privaten oder kollektiven Charakter hatten. Diese Unternehmen waren auf Märkte hin orientiert und intensi- vierten Schritt für Schritt den Wettbewerb auf den Gütermärkten. Alle Außenwirtschaftsbeziehungen und das gesamte Finanzsystem blieben jedoch vollständig reguliert. Durch seine Wechselkurspolitik sowie protektionistische Maßnahmen ist es China bis heute gelungen, eine über längere Zeiträume negative Leistungsbilanz zu verhindern. Die außenwirtschaftliche Verschuldung Chinas ist somit gering und entspricht in etwa den Währungsreserven der chinesischen Zentralbank. Insgesamt lassen sich die achtziger Jahre als langsames „Herauswachsen aus dem Plan“ charakterisieren.

$\mathrm{Ab}$ der Reformwelle Anfang der 90er Jahre setzte die chinesische Regierung verstärkt auf ausländische Direktinvestitionen, um möglichst schnell den technologischen Abstand zum Westen zu verkleinern. Ausländer können faktisch bis heute nur in der Form von Joint Ventures mit chinesischen Staatsunternehmen investieren, wobei ausländische Mehrheitsbeteiligungen ausgeschlossen sind. Trotz dieser Restriktionen erhielt China im letzten Jahrzehnt die höchsten Direktinvestitionen von allen unterentwickelten Ländern. Anfang der neunziger Jahre wurden zwei Börsen eröffnet, an der Minderheitsbeteiligungen von großen Staatsunternehmen gehandelt werden, die in Aktiengesellschaften umgewandelt wurden. In begrenztem Umfang wurde auch Ausländern der Kauf von Aktien chinesischer Unternehmen erlaubt, doch ist die Börse für In- und Ausländer strikt getrennt. Das Finanzsystem und die Außenwirtschaftsbeziehungen blieben in China auch in den 90er Jahren politisch reguliert.

Nicht nur mit der politischen Kontrolle der ökonomischen Entwicklung, auch mit seinem Gradualismus hebt sich China deutlich von den mittel- und osteu- 
ropäischen Transformationsländern $a b$, die zumindest bei den institutionellen Reformen in ihrer großen Mehrheit einer Schockstrategie gefolgt sind. Am härtesten wurde die Schockstrategie in den Ländern der ehemaligen Sowjetunion durchgeführt, was eine desaströse Entwicklung zur Folge hatte. In Russland halbierte sich nach 1989 das reale Sozialprodukt, die Ökonomie ist in die Bereiche Subsistenzwirtschaft, Tauschwirtschaft, Rubelwirtschaftszone und Devisenwirtschaftszone zerfallen. Kohärente ökonomische Bedingungen konnten nicht geschaffen werden und eine durchgreifende Besserung ist in Russland nicht in Sicht. Auch die mitteleuropäischen Transformationsländer mussten nach 1989 eine kräftige Schrumpfung des Sozialproduktes hinnehmen und konnten erst ab 1996 nach und nach das Produktionsniveau von 1989 erreichen. Demgegenüber ist es in China anscheinend gelungen, ein spezifisches Akkumulationsmodell zwischen Plan und Markt zu schaffen, das seine Dynamik zwanzig Jahre lang behielt. Zur Erklärung dieser langen Wachstumsphase vergleiche den Beitrag von Hansjörg Herr in diesem Heft.

IV.

Allerdings funktioniert auch dieses Akkumulationsmodell nicht ohne Schwierigkeiten und Widersprüche. Eines der chronischen Probleme ist die Korruption in der Form von Bestechung, illegalen Kreditmärkten und ausgeprägtem Schmuggel. Diese Phänomene sind zu einem guten Teil den engen staatlichen Regulierungen geschuldet, die zur Korruption geradezu einladen. Darüber hinaus spielen in China aber auch vielfältige Verhandlungsprozesse eine wichtige Rolle (zwischen Zentralstaat und Provinzen, zwischen Unternehmen, Banken und staatlichen Verwaltungen, zwischen verschiedenen Abteilungen von Unternehmen, zwischen Management und Arbeitnehmern etc., die in dieser Form in westlichen Ländern unbekannt sind) sowie „Beziehungen“, die eine breite Grauzone zwischen legalen und illegalen Prozessen ermöglichen. Korruption, Verhandlungslösungen und Beziehungsgeflechte sind integrale Bestandteile der chinesischen Ökonomie, die sich kurzfristig kaum ändern werden. Dementsprechend wenig Erfolg hatten denn auch die wiederholten Anti-Korruptionskampagnen der chinesischen Führung, bei der auch Todesurteile vollstreckt wurden.

Schon vor Beginn der Reformen 1978 und auch schon vor der Gründung der Volksrepublik gab es enorme Unterschiede zwischen Land und Stadt bzw. zwischen den stärker entwickelten Küstenprovinzen und dem Hinterland. Zwar wuchs seit 1978 der Lebensstandard in ländlichen Gebieten etwa mit der gleichen Rate wie in städtischen Gebieten, jedoch hat sich das Einkommensgefälle nicht eingeebnet, das Verhältnis der ProKopf-Einkommen zwischen Stadt und Land beträgt nach wie vor ca. 2,5:1. Wie sich das Stadt-Land-Gefälle auf die Lage der Frauen ausgewirkt hat, wird in dem Beitrag von Dagmar Yu-Dembski untersucht.

Bei den starken Einkommensunterschieden und regionalen Disparitäten ist es wenig verwunderlich, dass mehr und mehr Arbeitskräfte aus dem Hinterland in die Städte und die reichen Küstenprovinzen abwandern wollen. Das Ergebnis ist ein Anschwellen der sowieso im Vergleich zu Europa riesigen Städte mit enormen ökologischen und sozialen Problemen. Die Zuwanderer bilden in 
den Städten ein Segment äußerst niedrig bezahlter Arbeitskräfte, die im Vergleich zur „normalen“ Stadtbevölkerung über keinerlei soziale Absicherung verfügen. Sie verfügen über keine Arbeitslosenversicherung, keine Krankenversicherung, keinen Kündigungsschutz und keinen befriedigenden Wohnraum. Bauarbeiter schlafen auf den Baustellen, Arbeiterinnen in den Gaststätten, in denen sie tagsüber beschäftigt sind. Obdachlosigkeit wird zunehmend zum Problem. Für die politische Führung sind diese Entwicklungen kaum zu steuern, da sich die Arbeitskräfte über die gesetzlich nach wie vor existierenden Mobilitätsschranken hinwegsetzen und die Entwicklung des Hinterlandes trotz massiver Programme insbesondere im Infrastrukturbereich nicht schnell genug vorankommt. Erschwerend kommt hinzu, dass die reichen Küstenprovinzen wenig Neigung zeigen, eigene Steuermittel für die Entwicklung anderer Provinzen bereitzustellen und ein System des Finanzausgleichs zwischen den verschiedenen Provinzen nicht existiert.

Die regionalen Disparitäten werden auch durch die zunehmende Integration in den Weltmarkt verstärkt, ein Prozeß der sich durch den vor allem außenpolitisch motivierten Beitritt Chinas zur Welthandelsorganisation (WTO) noch verstärken dürfte. Sowohl die ausländischen Direktinvestitionen als auch die Masse der exportstarken Unternehmen konzentrieren sich auf die Küstenprovinzen. Typisch für diese Konzentration ist die Provinz Guangdong, die etwa 40\% der chinesischen Exporte auf sich vereinigt. Guangdong profitiert von der räumlichen Nähe zu Hongkong, das ökonomisch aufgrund einer eigenen Währung, Visumspflicht etc. auch nach seiner Rückgabe an China ökonomisch als
Ausland gelten muss. Eine wichtige Ursache der Entwicklung in Guangdong ist das große Kostengefälle zwischen Hongkong und dem Rest Chinas.

Die Integration Chinas als „verlängerte Werkbank“ wurde in erster Linie von Auslandschinesen betrieben. Direktinvestitionen westlicher Großunternehmen unterliegen einer anderen Logik. Diese Investitionen mussten die Form von Joint Ventures mit Staatsunternehmen annehmen. Arbeitsbedingungen, Sozialstandards und Bezahlung liegen in diesen Unternehmen über dem in China üblichen Niveau. Die chinesische Führung achtet bei diesem Typus ausländischer Investitionen auf den Technologietransfer und schreibt einen bestimmten Prozentsatz an „local content" vor. Für die westlichen Investoren sind die niedrigen chinesischen Löhne von zweitrangiger Bedeutung, ihnen geht es vor allem um den Zugang zum chinesischen Markt, dem ein enormes Entwicklungspotential zugetraut wird. Die Integration in den Weltmarkt und die daraus entstehenden ungleichgewichtigen Entwicklungen innerhalb Chinas stehen im Zentrum des Beitrags von Shaun Breslin.

\section{V.}

Bis jetzt ist es der Kommunistischen Partei Chinas gelungen, die Kontrolle über den wirtschaftlichen Transformationsprozeß zu behalten. Dieses Primat der Politik wird mit der immer weitergehenden Freisetzung der Marktkräfte aber zunehmend in Frage gestellt. Seit Mitte der neunziger Jahre gerät der Sektor der Staatsunternehmen beschleunigt in eine immer tiefere Krise. Auf einem immer wettbewerbsintensiveren Gütermarkt ist die früher sehr hohe Profitabilität der Staatsunternehmen aufgrund der Zerstörung von Monopolrenten drastisch ge- 
sunken. Die intensivere Konkurrenz auf den Gütermärkten ist das Resultat des Marktzutritts neuer Privatunternehmen sowie städtischer und ländlicher Kollektivbetriebe. Nicht unwesentlichen Anteil an dieser Entwicklung tragen auch die Joint Ventures, deren Bedeutung auf dem chinesischen Markt ständig zunimmt und die in aller Regel mit einer moderneren Technologie arbeiten und partiell durch Steuervorteile bevorzugt werden. Demgegenüber macht eine große Zahl von Staatsunternehmen Verluste und ist überschuldet. Die notleidenden Kredite der staatlichen Unternehmen belasten wiederum das Bankensystem. Die großen staatlichen Banken, die weit über zwei Drittel des Bankgeschäfts abwickeln, haben ein negatives Reinvermögen und können das Problem notleidender Kredite nicht aus eigener Kraft lösen. Da das Steuersystem nach planwirtschaftlicher Manier immer noch schwerpunktmäßig auf Zahlungen der Staatsunternehmen ruht, stecken die öffentlichen Haushalte in einer strukturellen fiskalischen Krise, die mit Ausgabenkürzungen (gemessen als Anteil am Sozialprodukt) beantwortet wurde. Verluste der Staatsunternehmen, notleidende Kredite und Krise des Steuerstaates haben den Druck auf die Regierung laufend erhöht. Als Ausweg wurde die Privatisierung verlustbringender Staatsunternehmen und eine Teilprivatisierung gewinnbringender Staatsunternehmen immer populärer. Raymond W.K. Lau arbeitet in seinem Beitrag den Druck zur Privatisierung sowie deren Mechanismen und Wirkungen heraus. Der kritischen Außensicht von Lau steht ein Beitrag von Zhongliang Shi gegenüber, der die offizielle chinesische Sichtweise der Probleme der Staatsunternehmen und die in China diskutierten Reformoptionen darstellt.
VI.

Obgleich China Schritt für Schritt in Richtung eines kapitalistischen Wirtschaftssystems gegangen ist und heute an der Schwelle zu einem vollständig entwickelten Kapitalismus steht, übt sich die Kommunistische Partei nach wie vor in einer sozialistischen Rhetorik. Im offiziellen Sprachgebrauch strebt China eine „sozialistische Marktwirtschaft” an. „Sozialismus" wird in China als Erhöhung des Lebensstandards der breiten Massen definiert, wobei es nicht weiter relevant sei, ob dieses Ziel über eine Markt- oder eine Planwirtschaft erreicht wird. Es ist egal, ob eine Katze weiß oder schwarz sei, war ein beliebter Ausspruch von Deng Xiaoping, Hauptsache sie fängt Mäuse. Die innerchinesische Diskussion offenbart somit eine erstaunliche Theorielosigkeit. Deng Xiaoping rückte dann auch die „Praxis“ der ökonomischen Veränderung ins Zentrum der politischen Debatte und kritisierte die Theorielastigkeit von Mao Zedong, die zu falschen Entwicklungen geführt habe.

Differenzierungen in den Lebensverhältnissen werden als notwendiges Übel des Entwicklungsprozesses hingenommen, doch bemüht sich die chinesische Führung darum, ein minimales soziales Netz $\mathrm{zu}$ erhalten und die Arbeitslosigkeit nicht eskalieren zu lassen. Allerdings ist es fraglich, wie lange eine solche Politik noch möglich sein wird. Die derzeit ablaufende Restrukturierung der Staatsunternehmen, unabhängig davon ob sie privatisiert werden oder nicht, wird zu Entlassungen großen Stils führen. Dasselbe gilt für die öffentlichen Verwaltungen. Da die sozialen Netze bisher vor allem von den Betrieben getragen worden ist, bleibt für die Entlassenen nicht mehr viel. Aber auch die Situation der noch Beschäftigten wird sich drastisch ändern, 
da zur Entlastung der Unternehmen und der öffentlichen Haushalte deren soziale Verpflichtungen deutlich abgebaut und die Lebensrisiken zunehmend individualisiert werden.

Es ist eine offene Frage, ob es der chinesischen Führung gelingt, die Probleme des staatlichen Unternehmenssektors, des Bankensystems sowie des Steuersystems so zu lösen, dass es auch weiterhin zu einem hohen Wachstum kommt, was dann allerdings mit noch weit größeren ökologischen Problemen verbunden wäre als dies ohnehin schon der Fall ist. Sollte es zu einer Reduzierung der Wachstumraten vom bisherigen Niveau von überzyklisch acht bis zehn Prozent auf nur drei oder vier Prozent kommen, würden Arbeitslosigkeit und die Probleme des staatlichen Sektors eskalieren. Auch schon eine kurzfristige Rezession hätte drastische Auswirkungen auf die soziale Lage in China. Aber selbst bei hohen Wachstumsraten sind Einschnitte ins soziale Netz unausweichlich und die bisherige Formel ,wachsender Wohlstand für alle gleich Sozialismus" wird nicht mehr aufrechtzuerhalten sein. Damit stellt sich die Frage, ob die Kommunistische Partei Chinas ihre bisherige politische Bindungskraft behaupten kann. Ohne eine auch ideologische Neuorientierung scheint dies ausgeschlossen. Kann Nationalismus, wie er etwa nach der Bombardierung der chinesischen Botschaft in Belgrad während des Kosovo-Krieges aufgebrandet ist, eine neue ideologische Klammer werden? Die Reibereien und das Säbelrasseln mit Taiwan könnten in diese Richtung deuten. Auch die Rivalität mit Japan um die Dominanz in Asien könnte nationalistische Strömungen stärken. Auf jeden Fall ist es wahrscheinlich, dass sich die Kommunistische Partei zunehmend von ihren sozialistischen Kompromissformeln lösen wird und dass China eher den Charakter einer kapitalistischen Entwicklungsdiktatur a la Südkorea annehmen wird. Mit dem Übergang zum Kapitalismus wird China auch mit allen Problemen dieser Produktionsweise konfrontiert werden, was über kurz oder lang auch entsprechende Veränderungen des politischen Systems und seiner Legitimationsideologie nach sich ziehen muß.

$$
* * *
$$

Die verschiedenen Beiträge über die kapitalistische Entwicklung Chinas werden durch Markus Pohlmanns kritische Auseinandersetzung mit der „neuen Konfuzianismusthese" ergänzt, welche die bekannte Position Max Webers gewissermaßen umdrehte: aus einem Entwicklungshindernis sollte der Konfuzianismus nun zum Motor einer kapitalistischen Entwicklung Asiens geworden sein.

Außerhalb des Schwerpunkts erscheint ein Beitrag von Raimund Feld zur Entwicklung der schwedischen Sozialdemokratie in den vergangenen beiden Jahrzehnten. Darin wird aufgezeigt wie Schweden, das für die reformistische Linke Europas lange Zeit als Vorbild galt, vor allem von sozialdemokratischen Regierungen in Richtung einer neoliberalen Entwicklung geführt wurde. 